\title{
ANÁLISE DO EFEITO DA SAZONALIDADE SOBRE O RENDIMENTO DO ÓLEO ESSENCIAL DAS FOLHAS DE Nectandra grandiflora Nees ${ }^{1}$
}

\author{
Daniela Thomas da Silva ${ }^{2}$, Nadia Helena Bianchini ${ }^{3}$, Lúcio de Paula Amaral ${ }^{4}$, Solon Jonas Longhi ${ }^{5}$ e Berta
} Maria Heinzmann 6

\begin{abstract}
RESUMO - Este estudo teve por objetivos avaliar o efeito da sazonalidade sobre o teor de óleo essencial (OE) presente nas folhas de Nectandra grandiflora Nees e determinar se a secagem do material vegetal à temperatura ambiente influencia o rendimento do OE. Para tanto, foi coletado material vegetal de 12 indivíduos ao longo de um ano, e o $\mathrm{OE}$ foi extraído separadamente de folhas frescas e folhas secas, pelo método de hidrodestilação. Paralelamente, $20 \mathrm{~g}$ de folhas frescas foram levados à estufa a $40{ }^{\circ} \mathrm{C}$ até peso constante, para determinação do teor de umidade, procedimento esse realizado para cada coleta, individualmente. $\mathrm{O}$ rendimento de $\mathrm{OE}$ das folhas $(\% \mathrm{~m} / \mathrm{m})$ foi calculado para o material vegetal fresco com base em massa de matéria seca, determinado considerando o teor de umidade, bem como para o material seco à temperatura ambiente. Os dados de rendimento foram analisados através do teste de Tukey $(P<0,05)$. Os resultados mostraram que o teor de OE das folhas da espécie apresentou variabilidade sazonal, com a maior produção detectada na primavera $(0,75 \pm 0,06 \%)$. Esse valor foi estatisticamente diferente dos rendimentos encontrados nas outras estações do ano, sendo o menor rendimento obtido no inverno $(0,39 \pm 0,02 \%)$. Desse modo, indicase que a coleta de folhas de $N$. grandiflora seja realizada durante a primavera, visando obter maior teor de $\mathrm{OE}$. Adicionalmente, verificou-se que não há perda de teor $\mathrm{OE}$ durante o processo de secagem à temperatura ambiente e, dessa forma, esse procedimento pós-colheita poderá vir a ser adotado sem comprometer o rendimento do extrativo.
\end{abstract}

Palavras-chave: Extrativos vegetais; Lauraceae; Produtos florestais não madeireiros.

\section{ANALYSIS OF THE SEASONALITY EFFECT ON THE ESSENTIAL OIL YIELD OF LEAVES OF Nectandra grandiflora Nees}

\begin{abstract}
This study aimed to evaluate the effect of seasonal variability on the yield of essential oil (EO) present in leaves of Nectandra grandiflora Nees and as well as to determine whether the drying process at room temperature of the plant material influences the yield of this EO. Plant material was collected from 12 individuals over a year and $E O$ was extracted by hydrodestillation separately from fresh and dried leaves. Simultaneously, $20 \mathrm{~g}$ of fresh leaves was taken to stove at $40{ }^{\circ} \mathrm{C}$ to constant weight for determination of moisture content. This procedure was performed for each collection individually. The yield of EO in leaves (\% w/w) was calculated for fresh plant material based on dry matter, determined by considering the moisture content, as well as for the material dried at room temperature. The yields were analyzed by Tukey test $(\mathrm{P}<0.05)$. Results
\end{abstract}

\footnotetext{
${ }^{1}$ Recebido em 04.02.2013 aceito para publicação em 13.10.2015.

${ }^{2}$ Universidade Federal de Santa Maria, UFSM, Programa de Pós-graduação em Engenharia Florestal, Santa Maria, RS - Brasil. E-mail: <dthomasdasilva@gmail.com>.

${ }^{3}$ Universidade Federal de Santa Maria, Graduado em Engenharia Florestal, Santa Maria, RS - Brasil. E-mail: $<$ nadia_bianchini@hotmail.com>.

${ }^{4}$ Universidade Federal de Santa Maria, Centro de Ciências Rurais, Departamento de Engenharia Rural, Santa Maria, RS Brasil. E-mail: <lpamaralengflorestal@gmail.com>.

${ }^{5}$ Universidade Federal de Santa Maria, Centro de Ciências Rurais, Departamento de Ciências Florestais, Santa Maria, RS - Brasil. E-mail: <longhi.solon@gmail.com>.

${ }^{6}$ Universidade Federal de Santa Maria, Centro de Ciências da Saúde, Departamento de Farmácia Industrial, Santa Maria, RS - Brasil. E-mail: <berta.heinzmann@gmail.com>.
} 


\begin{abstract}
showed that the content of EO in the leaves showed seasonal variability with the highest production detected in spring $(0.75 \pm 0.06 \%)$ and the lowest yield in winter $(0.39 \pm 0.02 \%)$. Thereby, it is recommended that the leaves of $\mathbf{N}$. grandiflora be collected in spring, aiming to increase EO yield. Additionally, no loss of EO content during the drying process at room temperature occurred thus this postharvest procedure could be adopted without compromising the extractive yield.
\end{abstract}

Keywords: Plant extracts; Lauraceae; Non-timber forest products.

\section{INTRODUÇÃO}

As espécies fornecedoras de produtos florestais não madeireiros, como aquelas que produzem óleo essencial (OE), têm sido reconhecidas como fonte alternativa de renda e importante ferramenta para conservação e manejo dos recursos florestais (TORRES, 2001; BATISH et al., 2008). As plantas aromáticas podem alcançar alto valor no mercado (MARQUES, 2001), além de contribuírem para o conhecimento do potencial econômico dos biomas, visando à utilização sustentável de seus recursos.

Desde a Antiguidade, os OE, também conhecidos como óleos voláteis ou essências, são amplamente utilizados devido às suas propriedades antifúngicas, antibacterianas, antiparasitárias e inseticidas. Atualmente, são conhecidos aproximadamente $3.000 \mathrm{OE}$, sendo 300 comercialmente importantes. Isso se deve ao fato de possuírem aplicação na perfumaria, em produtos de limpeza, na cosmética, conservação de alimentos, como coadjuvantes de medicamentos e na indústria farmacêutica (BAKKALI et al., 2008; BIZZO et al., 2009).

Nectandra grandiflora Nees (Lauraceae), conhecida popularmente como canela-amarela, é espécie endêmica do Brasil, encontrada predominantemente nos biomas Mata Atlântica e Cerrado (LORENZI, 2002). Na medicina popular, $N$. grandiflora é utilizada como antirreumática, diurética e digestiva (RAGGI, 2008). Os estudos farmacológicos realizados com o $\mathrm{OE}$ das folhas dessa espécie são poucos e indicam potencial antimicrobiano (CUNICO et al., 2010).

Os constituintes voláteis dos OE são produzidos por células secretoras especializadas que minimizam o risco de autotoxicidade e, simultaneamente, permitem a presença de concentrações elevadas de metabólitos secundários em locais onde sua função de defesa e/ ou atração pode ser vital (FIGUEIREDO et al., 2008). Há inúmeros fatores que podem levar a variações na quantidade e constância da composição de metabólitos secundários, os quais incluem as substâncias de baixo peso molecular e alta volatilidade que compõem os
OE. Entre esses fatores, destaca-se a sazonalidade, termo utilizado para designar as variações que ocorrem em decorrência das diferentes estações do ano. Essas variações estão relacionadas, principalmente, com fatores climáticos e ambientais. No entanto, outros fatores também podem influenciar tanto quali quanto quantitativamente a produção de metabólitos secundários, como as características genéticas da espécie, fatores inerentes à própria planta, como idade e ciclo de desenvolvimento, os macro e micronutrientes do solo, a altitude, longitude e a ocorrência de predadores ou patógenos, poluição atmosférica etc. Desse modo, fica clara a necessidade de estudos que avaliem as condições e épocas para coleta, levando, assim, a uma elevada produção de matéria-prima de qualidade (GOBBONETO; LOPES, 2007; FIGUEIREDO et al., 2008). Considerando os aspectos anteriormente expostos, este estudo visou analisar o efeito da sazonalidade sobre o rendimento de OE das folhas de $N$. grandiflora, buscando definir a época de colheita do material vegetal em função da maior produção de compostos voláteis. Adicionalmente, buscou-se determinar se há diminuição dos teores de OE após a secagem do material, com o objetivo de avaliar o possível uso desse procedimento pós-colheita.

\section{MATERIAL E MÉTODOS}

\subsection{Obtenção do material vegetal}

O material vegetal de $N$. grandiflora Nees foi coletado em população nativa, em área no Município de Jaguari, Rio Grande do Sul, localizada nas coordenadas geográficas $-29^{\circ} 26^{\prime} 25,09^{\prime \prime}$ de latitude e -54²40’27,73" de longitude. A espécie foi identificada pelo Dr. Solon Jonas Longhi, e uma exsicata de registro SMDB 13.162 encontra-se arquivada no Herbário do Departamento de Biologia, da Universidade Federal de Santa Maria (UFSM). Para a avaliação do rendimento sazonal do OE, foram coletadas folhas de 12 indivíduos (três indivíduos em cada estação) da espécie entre os meses de julho de 2011 e junho de 2012 . 


\subsection{Determinação do teor de umidade}

Para determinação da umidade, após a retirada das folhas dos galhos e homogeneização do material vegetal, preparou-se uma amostra de aproximadamente $20 \mathrm{~g}$ de folhas frescas de cada indivíduo, as quais, em seguida, foram submetidas à secagem em estufa a $40^{\circ} \mathrm{C}$ até peso constante. $\mathrm{O}$ teor de umidade presente em cada amostra foi calculado através da fórmula $\frac{\text { Púmido - Pseco }}{\text { Púmido }} \times 100$. A fím de comprovar a influência do teor de água sobre o teor de OE, utilizaram-se, para os cálculos de rendimento, os valores de massa das folhas frescas em relação à massa fresca (MF BU) e à massa seca (MF BS) do material vegetal. A massa foliar à base seca (MF BS) foi corrigida pelo teor de umidade presente no material vegetal, através da fórmula $M F B U-\frac{T U-M F B U}{100}$, em que MF BU é equivalente à massa das folhas frescas usadas nas extrações dos $\mathrm{OE}$.

\subsection{Obtenção do óleo essencial}

O material vegetal picado, composto de folhas frescas e folhas secas, foi submetido separadamente à hidrodestilação, utilizando-se o aparelho Clevenger modificado (FARMACOPEIA BRASILEIRA, 2010), acoplado a um balão de fundo redondo, com capacidade para $5 \mathrm{~L}$, durante $3 \mathrm{~h}$. As extrações foram realizadas em triplicatas, com aproximadamente $500 \mathrm{~g}$ de folhas frescas ou $300 \mathrm{~g}$ de folhas secas em cada balão. Para a obtenção das folhas secas, o material vegetal correspondente foi submetido à secagem branda logo após a coleta, sem aquecimento nem circulação de ar, ou seja, as folhas foram secas à temperatura ambiente e à sombra, durante 15 dias. O teor de OE foi obtido por pesagem em balança analítica e posterior cálculo do rendimento $(\% \mathrm{~m} / \mathrm{m})$, em relação à massa foliar à base úmida (MF BU), massa foliar à base seca (MF $\mathrm{BS}$ ) e massa foliar seca à temperatura ambiente (FS). Os OE obtidos foram armazenados em frascos de vidro âmbar, vedados e conservados à $-4{ }^{\circ} \mathrm{C}$.

\subsection{Análise estatística}

Os tratamentos avaliados foram as matérias-primas fresca e seca à temperatura ambiente. A variável analisada foi o rendimento de $\mathrm{OE}(\mathrm{m} / \mathrm{m} \%)$ transformado para arcosseno $\frac{\sqrt{\mathrm{R} \%}}{100}$ anteriormente à análise estatística. Para verificação da normalidade dos dados e homogeneidade de variâncias, os dados de rendimento obtidos foram submetidos aos testes de ShapiroWilk e Levene, respectivamente. A análise de variância (ANOVA) de uma via, os testes de Tukey, MannWhitney e teste $t$ foram usados para comparar os dados de rendimento, apresentados por média \pm EPM. As análises foram realizadas no software SigmaPlot, versão 11.0 , com o nível mínimo de significância $(P<0,05)$ (PIMENTEL et al., 2012; AMARAL et al., 2015).

\section{RESULTADOS}

O rendimento médio obtido do $\mathrm{OE}$ das folhas frescas (MF BS) de $N$. grandiflora no referido ano de coleta foi igual a $0,54 \pm 0,03 \%$, enquanto nas folhas secas o rendimento médio correspondente foi de $0,51 \pm 0,02 \%$. O OE extraído das folhas frescas (MF BS) de $N$. grandiflora apresentou variabilidade sazonal significativa em relação ao rendimento, segundo o teste de Tukey. Os indivíduos coletados durante a primavera apresentaram teores significativamente maiores em comparação com os indivíduos coletados nas demais estações do ano. Cabe ressaltar que não houve diferença estatística entre

Diferentes letras minúsculas indicam diferenças significativas entre os rendimentos, pelo teste de Tukey $(P<0,05)$.

Different lowercase letters indicate significant differences between yields, based on Tukey test $(P<0.05)$.

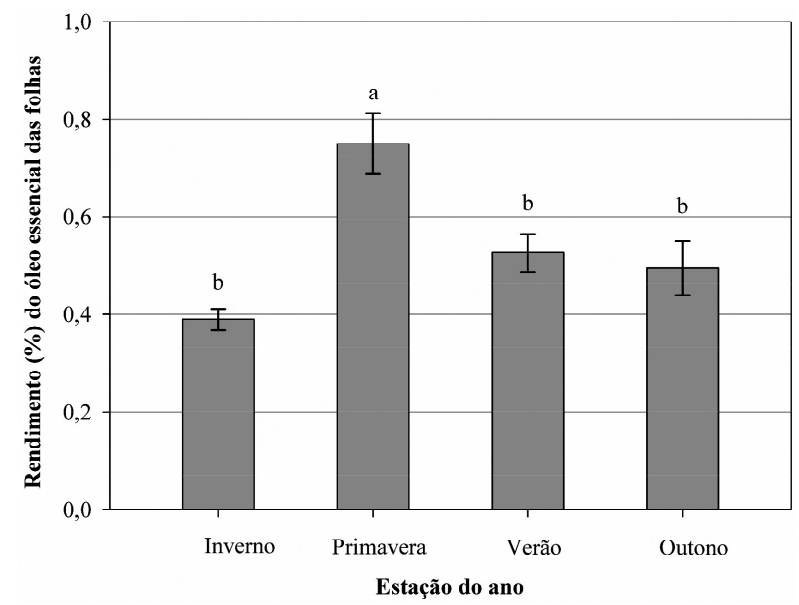

Figura 1 - Rendimentos médios (\%) dos óleos essenciais das folhas frescas (MF BS) obtidas de 12 indivíduos de Nectandra grandiflora nas quatro estações do ano (três coletas por estação).

Figure 1 - Average yields (\%) of essential oils of fresh leaves (MF BS) obtained from 12 individuals of Nectandra grandiflora in four seasons (three collections per season).

Revista Árvore, Viçosa-MG, v.39, n.6, p.1065-1072, 2015

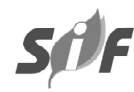


os rendimentos de OE resultantes das coletas realizadas durante o verão, outono e inverno (Figura 1).

A quantidade de OE armazenado nas folhas frescas (MF BS) de $N$. grandiflora apresentou rendimento máximo de $0,75 \pm 0,06 \%$ na primavera, período que coincide com a floração da planta e com o maior nível de umidade $(49,09 \%)$. Durante o inverno, foi verificado menor rendimento de OE nas folhas frescas (MF BS), igual a $0,39 \pm 0,02 \%$, período em que o teor de umidade das folhas também foi o mais baixo (40,76\%) (Figura 2).

A fim de avaliar a influência da secagem branda das folhas de N. grandiflora, à temperatura ambiente, sobre o teor de OE, os rendimentos obtidos das folhas secas (FS) foram comparados com aqueles obtidos das folhas frescas, corrigidos pela umidade (MF BS). Os resultados não apresentaram diferença significativa entre os rendimentos avaliados de cada estação do ano. Desse modo, as folhas submetidas à secagem não sofreram influência negativa desse processamento póscolheita em relação ao teor de OE presente no referido órgão vegetal. Houve somente a perda de água, ou seja, perda por dessecação das folhas, sem afetar a quantidade de $\mathrm{OE}$.

Realizou-se, paralelamente, a contraprova desse resultado, em que foram avaliados estatisticamente os dados obtidos dos rendimentos de massa fresca em base úmida (MF BU) em relação aos demais rendimentos calculados (MF BS e FS). As análises estatísticas demonstraram que os rendimentos de MF BU são diferentes estatisticamente daqueles obtidos a partir de MF BS e FS, em cada período sazonal (Figura 3 ). Desse modo, foi confirmada a influência do teor de água presente nas folhas sobre o teor final de OE, sendo indicada a retirada da massa de água dos cálculos e avaliações de rendimento.

\section{DISCUSSÃO}

$\mathrm{O}$ rendimento médio anual à base seca do $\mathrm{OE}$ das folhas frescas (MF BS) de $N$. grandiflora verificado no ano de referência foi inferior ao encontrado em espécies de outros gêneros da família Lauraceae, considerando-se o mesmo órgão vegetal. A espécie Ocotea porosa (Nees \& Mart.) Barroso apresentou teor de $0,82 \%$ (BRITO, 2009), enquanto Taveira et al. (2003) obtiveram rendimentos entre 0,5-0,8\% para Aniba canelilla (Kunth) Mez. Já O. quixos (Lam) Kosterm teve teor de OE equivalente a 1,6\% (SACCHETTI et al., 2006). No entanto, em comparação com outras espécies de Nectandra, verificou-se que o OE obtido da canelaamarela apresentou rendimento superior ao da $N$. salicina C. K. Allen e ao da N. megapotamica (Spreng.) Mez (APEL et al., 2006; CICCIO et al., 2009).

O efeito da sazonalidade sobre o rendimento de OE também foi verificado em espécies como $O$. porosa e N. megapotamica, A. canelilla, O. odorifera (Vell.) Rohwer, Corymbia citriodora (Hook.) K.D. Hill \& L.A.S. Johnson e Siparuna guianensis Aubl. (VITTI; BRITO; 1999; TAVEIRA et al., 2003; CASTELANI et al., 2006; BRITO, 2009; VALENTINI et al., 2010; AMARAL et

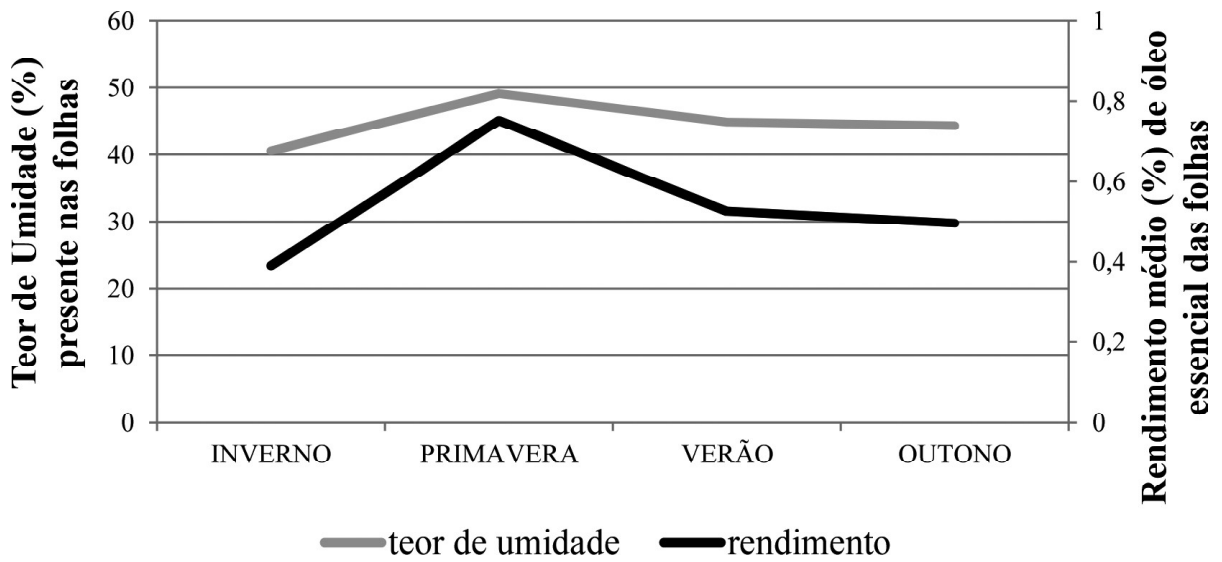

Figura 2 - Representação gráfica da variação sazonal do rendimento do óleo essencial das folhas frescas (MF BS) de Nectandra grandiflora e do teor de umidade presente no mesmo material vegetal.

Figure 2-Graphical representation of seasonal variability of essential oil yields of fresh leaves (MF BS) of Nectandra grandiflora and the moisture content present in the same plant material.

Revista Árvore, Viçosa-MG, v.39, n.6, p.1065-1072, 2015 
Diferentes letras maiúsculas indicam diferenças significativas de rendimento entre os grupos e diferentes letras minúsculas indicam diferenças significativas entre os rendimentos dentro do mesmo grupo, pelo teste de Tukey $(P<0,05)$.

Different uppercase letters indicate significant differences of yields between groups and different lowercase letters indicate significant differences between yields of the same group, based on Tukey test $(P<0.05)$.

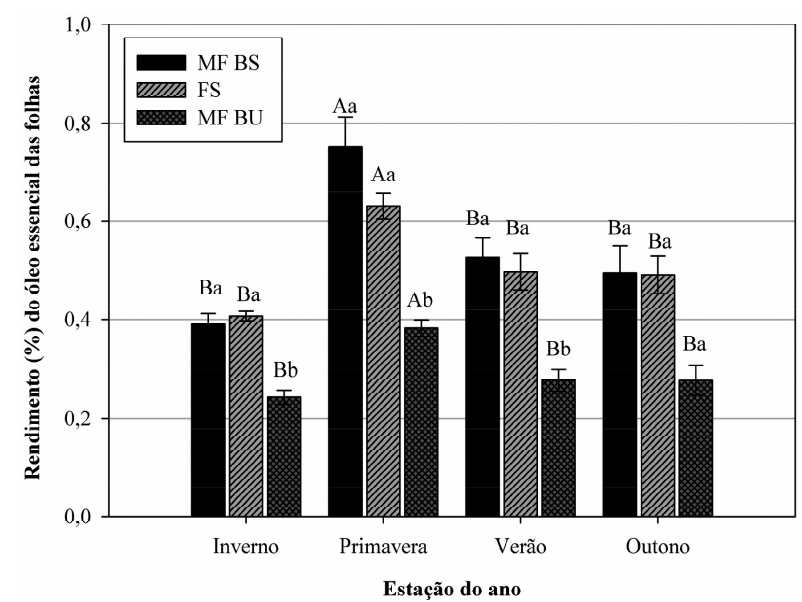

Figura 3 - Rendimentos (\%) dos óleos essenciais das folhas frescas determinados à base seca (MF BS) e à base úmida (MF BU) e das folhas secas (FS) de Nectandra grandiflora nas quatro estações do ano.

Figure 3 - Yields (\%) of essential oils of fresh leaves based on dry (MF BS) and fresh weight (MF BU), and of dry leaves (DL) of Nectandra grandiflora in four seasons.

al., 2015). Nesses estudos, observou-se que a quantidade e composição são, muitas vezes, voláteis e dependentes da espécie e das fases fenológicas em que ela se encontra. Conforme Gobbo-Neto e Lopes (2007), a tendência no aumento do rendimento dos OE é mais suscetível ao ciclo vegetativo que as variações climáticas observadas. Vale ressaltar que a espécie $N$. megapotamica, que também ocorre no bioma Mata Atlântica, teve a influência sazonal sobre a produção de OE recentemente avaliada e, da mesma forma que $N$. grandiflora, apresentou maior teor de OE na primavera (AMARAL et al., 2015).

Pesquisas fenológicas com $N$. grandiflora realizadas no Sul do Brasil confirmam que a espécie floresce de agosto a outubro (CARVALHO, 1980; MARCHIORI, 1997). O período de floração coincidiu com os maiores teores de OE das folhas de $N$. grandiflora, ou seja, houve aumento significativo no teor de OE durante a estação da primavera. A variabilidade sazonal encontrada no OE das folhas de $N$. grandiflora é similar à observada nas partes aéreas de Baccharis dracuncifolia DC. e B. uncinella DC. Estudos de Frizzo et al. (2008) indicam que a coleta de ambas as espécies deve ocorrer durante o estágio de plena floração. No entanto, nesses casos o processo de coleta deve ser realizado de modo a minimizar os prejuízos sobre a reprodução da espécie, que inclui, principalmente, eventos como floração, polinização e frutificação. Além disso, deve-se tomar o cuidado para que seja realizada uma seleção de diferentes árvores matrizes a cada ano de coleta e proceder, periodicamente, a um controle qualitativo dos extratos vegetais.

Adicionalmente, foi observado que os níveis de umidade presentes nas folhas foram diretamente proporcionais aos teores de OE no mesmo material vegetal durante o período de estudo. Desse modo, há indícios de que o rendimento de OE possa estar ligado também à disponibilidade hídrica do solo, como detectado em O. odorifera e Casearia sylvestris Sw. (CASTELANI et al., 2006).

Uma das possíveis explicações para a permanência de compostos voláteis nas folhas de $N$. grandiflora, mesmo após sua secagem, relaciona-se à morfoanatomia das suas folhas. As substâncias lipofílicas e mucilaginosas presentes em espécies da família Lauraceae são secretadas por estruturas internas denominadas idioblastos, que são encontrados em meio ao parênquima paliçádico e/ou esponjoso (FARAGO et al., 2005; COUTINHO et al., 2006; FIGUEIREDO et al., 2008; MARANHO et al., 2009). Os idioblastos secretores se distinguem das estruturas multicelulares pelo conteúdo, forma, tamanho e espessamento da parede (FARAGO et al., 2005), motivo pelo qual o OE contido nessas estruturas está protegido, e, assim, a perda por volatilização durante o processo de secagem não ocorre. Ao contrário, os OE contidos em estruturas secretoras externas como os tricomas glandulares, dependendo do método de secagem, são fortemente afetados (VENSKUTONIS, 1997; SELLAMI et al., 2012).

Estudos da literatura indicam, no entanto, que, mesmo que os OE estejam armazenados em células internas, a análise de métodos alternativos de secagem deve ser realizada (COSTA et al., 2005; PIMENTEL et al., 2012; RAHIMMALEK; GOLI, 2013). Costa et al. (2005) relataram o uso do desumidificador como método eficiente para a secagem do material vegetal antes da

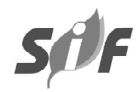

Revista Árvore, Viçosa-MG, v.39, n.6, p.1065-1072, 2015 
extração do OE, visando manter as características originais de Cymbopogon citratus (DC.) Stapf. Entretanto, Rahimmalek e Goli(2013) indicaram que a secagem através de micro-ondas é um método mais rápido, além de envolver menores custos.

\section{CONCLUSÃO}

A espécie Nectandra grandiflora apresentou variação sazonal do rendimento do óleo essencial das folhas, o que pode ter ocorrido em função do período fenológico e do teor de umidade do material vegetal. O melhor rendimento foi obtido durante a primavera, época de floração da espécie. Uma vez que não houve perda de óleo essencial através do processo de secagem branda, o material vegetal pode ser submetido a esse processamento pós-colheita sem comprometer o rendimento do extrativo. No entanto, pesquisas envolvendo a composição química do óleo essencial, bem como a comparação entre diferentes métodos de secagem, devem ser realizadas a fim de otimizar a obtenção de óleo essencial de folhas de Nectandra grandiflora.

\section{AGRADECIMENTOS}

À Fundação de Amparo à Pesquisa do Estado do Rio Grande do Sul (FAPERGS/PRONEX, Documento ${ }^{\circ}$. 10/0016-8), Conselho Nacional de Pesquisa e Desenvolvimento Científico (CNPq, Documento $\mathrm{n}^{\circ}$. 470964/2009-0) e à Coordenação de Aperfeiçoamento de Pessoal de Nível Superior (CAPES), pelas bolsas concedidas. Ao EngenheiroAgrônomo Jorge Diefenthaeler, pela colaboração na coleta dos dados.

\section{REFERÊNCIAS}

AMARAL, L.P.; TONDOLO, J.S.M.; SCHINDLER, B.; SILVA, D.T.; PINHEIRO, C.G.; LONGHI, S.J.; MALLMANN, C.A.; HEINZMANN, B.M. Seasonal influence on the essential oil production of Nectandra megapotamica (Spreng.) Mez. Brazilian Archives of Biology and Technology, v.58, n.1, p.12-21, 2015.

APEL, M.A.; LIMA, M.E.L.; SOUZA, A.; CORDEIRO, I.; YOUNG, M.C.M; SOBRAL, M.E.G.; SUFFREDINI, I.B.; MORENO, P.R.H. Screening of the biological activity from essential oils of native species from Atlantic rain forest (São Paulo - Brazil). Pharmacologyonline, v.3, p.376-383, 2006.

BAKKALI, F.;AVERBECK, S.; AVERBECK, D.; IDAOMAR, M. Biological effects of essential oils A Review. Food and Chemical Toxicology, v.46, n.2, p.446-475, 2008.

BATISH, D.R.; SINGH, H.P.; KOHLI, R.K.; KAUR, S. Eucalyptus essential oil as a natural pesticide.

Forest Ecology and Management, v.256, n.12, p.2166-2174, 2008.

BIZZO, H.R.; HOVELL, A.M.C.; REZENDE, C.M. Óleos essenciais no Brasil: aspectos gerais, desenvolvimento e perspectivas Química Nova, v.32, n.3, p.588-594, 2009.

BRITO, A.F.R. Análise da variação sazonal e das atividades antifúngica e antimicrobiana em óleos essenciais de Ocotea porosa (Nees) Barroso e Nectandra megapotamica (Spreng.) Mez. 2009. $261 \mathrm{f}$. Dissertação (Mestrado em Química Orgânica) Universidade de São Paulo, São Paulo, 2009.

CARVALHO, P.E.R. Levantamento florístico da região de Irati-PR ( $1^{\mathrm{a}}$ aproximação). Curitiba: Embrapa, 1980. (Circular Técnica, 3)

CASTELLANI, D.C; CASALI, V.W.D.; SOUZA, A.L.; CECON, P.R.; CARDOSO, C.A.; MARQUES, V.B. Produção de óleo essencial em canela (Ocotea odorifera Vell) e guaçatonga (Casearia sylvestris Swartz) em função da época de colheita. Revista Brasileira de Plantas Medicinais, v.8, n.4, p.104-107, 2006.

CICCIÓ, J.F.; CHAVERRI, C.; DÍAZ, C. Volatile compounds of Nectandra salicina (Lauraceae) from Costa Rica and their cytotoxic activity on cell lines. Química Nova, v.32, n.2, p.417-420, 2009.

COSTA, L.C.B.; CORRÊA, R.M.; CARDOSO, J.C.W.; PINTO, J.E.B.P.; BERTOLUCCI, S.K.V.; FERRI, P.H.

Secagem e fragmentação da matéria seca no rendimento e composição do óleo essencial do capim-limão. Horticultura Brasileira, v.23, n.4, p.956-959, 2005.

COUTINHO, D.F.; MAGRA, M.F.; BARBOSAFILHO, J.M.; BASÍLIO, I.J.L.D. Morfo-anatomia foliar de Ocotea gardneri (Meisn.) Mez 
(Lauraceae-Lauroideae). Brazilian Journal of Pharmacognosy, v.16, n.2, p.178-184, 2006.

CUNICO, M.M.; VIEIRA, G., LIMA, C.P.; CÔCCO, L.C.; YAMAMOTO, C.I.; AUER, C.G.; SANQUETTA, C.R. Estudo preliminar antimicrobiano e fitoquímico do óleo essencial foliar da Nectandra grandiflora Nees (canelaamarela). In: INTERNATIONAL CONFERENCE ON EDUCATION FOR SUSTAINABLE DEVELOPMENT, 2010, Curitiba. Anais... Curitiba: UFPR, 2010.

FARMACOPEIA BRASILEIRA. 5.ed. São Paulo: Atheneu, 2010.

FARAGO, P.V.; BUDEL, J.M.; DUARTE, M.R.; NAKASHIMA, T. Análise morfoanatômica de folhas de Ocotea puberula (Rich.) Nees, Lauraceae. Brazilian Journal of Pharmacognosy, v. 15, n.3, p.250-255, 2005.

FIGUEIREDO, A.C.; BARROSO, J.G.; PEDRO, L.G.; SCHEFFER, J.J.C. Factors affecting secondary metabolite production in plants: volatile components and essential oils. Flavour and Fragrance Journal, v.23, n.1,p.213-226, 2008

FRIZZO, C.D.; ATTI-SERAFINI, L.; LAGUNA, S.E.; CASSEL, E.; LORENZO, D.; DELLACASA, E. Essential oil variability in Baccharis uncinella DC and Baccharis dracunculifolia DC growing wild in southern Brazil, Bolivia and Uruguay. Flavour and Fragrance Journal, v.23, n. 1, p.99-106, 2008.

GOBBO-NETO, L.; LOPES, N.P. Plantas medicinais: fatores de influência no conteúdo de metabólitos secundários. Química Nova, v.30, n.2, p.374-381, 2007.

SELLAMI, I.H.; REBEY, I.B.; SRITI, J.; RAHALI, F.Z.; LIMAM, F.; MARZOUK, B. Drying sage (Salvia officinalis L.) plants and its effects on content, chemical composition, and radical scavenging activity of the essential oil. Food and Brioprocess Technology, v.5, n.8, p.2978-2989, 2012.

LORENZI, H. Árvores brasileiras: manual de identificação e cultivo de plantas arbóreas nativas do Brasil. Nova Odessa: Plantarum, 2002. v.2.
MARANHO, L.T.; PREUSSLER, K.H.; ROCHA, L.D. Organização estrutural da casca de Persea major Kopp (Lauraceae). Acta Botanica Brasilica, v.23, p.509-515, 2009.

MARCHIORI, J.N.C. Dendrologia das angiospermas: das magnoliáceas às flacurtiáceas. Santa Maria: UFSM, 1997.

MARQUES, C.A. Importância da família Lauraceae Lindl. Floresta e Ambiente, v.8, n.1, p.195206, 2001.

PIMENTEL, F.A.; CARDOSO, M.G.; ANDRADE, M.A.; ZACARONI, L.M.; GUIMARÃES, L.G.L. Influência da secagem sobre o rendimento e composição química dos compostos voláteis das raízes de Piper piscatorum Trel. \& Yunck. (Piperaceae). Química Nova, v.35, n.4, p.715$718,2012$.

RAGGI, L. Estudo da composição química e das atividades biológicas dos óleos voláteis de espécies de Lauraceae, em diferentes épocas do ano. 2008. 67f. Dissertação (Mestrado em Biodiversidade Vegetal e Meio Ambiente) Instituto de Botânica da Secretaria de Estado do Meio Ambiente, São Paulo, 2008.

RAHIMMALEK, M.; GOLI, S.A.H. Evaluation of six drying treatments with respect to essential oil yield, composition and color characteristics of Thymys daenensis subsp. daenensis. Celak leaves. Industrial Crops and Products, v.42, p.613-619, 2013.

SACCHETI, G.; GUERRINI, A.; NORIEGA, P.; BIANCHI, A.; BRUNI, R. Essential oil of wild Ocotea quixos (Lam.) Kosterm. (Lauraceae) leaves from Amazonian Ecuador. Flavour and Fragrance Journal, v.21, n.5, p.674-676, 2006.

TAVEIRA, F.S.N.; LIMA, W.N.; ANDRADE, E.H.A.; MAIA, J.G.S. Seasonal essential oil variation of Aniba canelilla. Biochemical Systematics and Ecology, v.31, n.1, p.69$75,2003$.

TORRES, M.R. Compilación y análisis sobre los productos forestales no madereros (PFNM) en el Perú. 2001. 59p. San Tiago: FAO, 2001 (Estudios nacionales

Revista Árvore, Viçosa-MG, v.39, n.6, p.1065-1072, 2015 
sobre productos no madereros en América Latina - GCP/RLA/133/EC)

VALENTINI, C.M.A.; SILVA, L.E.; MACIEL, E.N.; FRANCESCHINI, E.; SOUSA JR., P.T.;

DALL'OGLIO, E.L.; COELHO, M.F.B. Variação anual do rendimento e composição química dos componentes voláteis da Siparuna guianensis Aublet. Química Nova, v.33, n.7, p.1506-1509, 2010.
VENSKUTONIS, P.R. Effect of drying on the volatile constituents of thyme (Thymus vulgaris L.) and sage (Salvia oficinalis L.). Food Chemistry, v.59, n.2, p.219-227, 1997.

VITTI, A.M.S.; BRITO, J.O. Avaliação do rendimento e do teor de citronelal do óleo essencial de procedências e raças locais de Eucalyptus citriodora. Scientia Florestalis, n.56, p.145-154, 1999. 